\title{
Charge carrier dynamics in semiconducting mercury cluster anions
}

\author{
Ryan M Young ${ }^{1}$, Graham B Griffin ${ }^{1}$, Oli T Ehrler ${ }^{1}$, Aster Kammrath ${ }^{1,2}$, \\ Arthur E Bragg ${ }^{1,3}$, Jan R R Verlet ${ }^{1,4}$, Ori Cheshnovsky ${ }^{5}$ and \\ Daniel M Neumark ${ }^{1,3,6}$
}

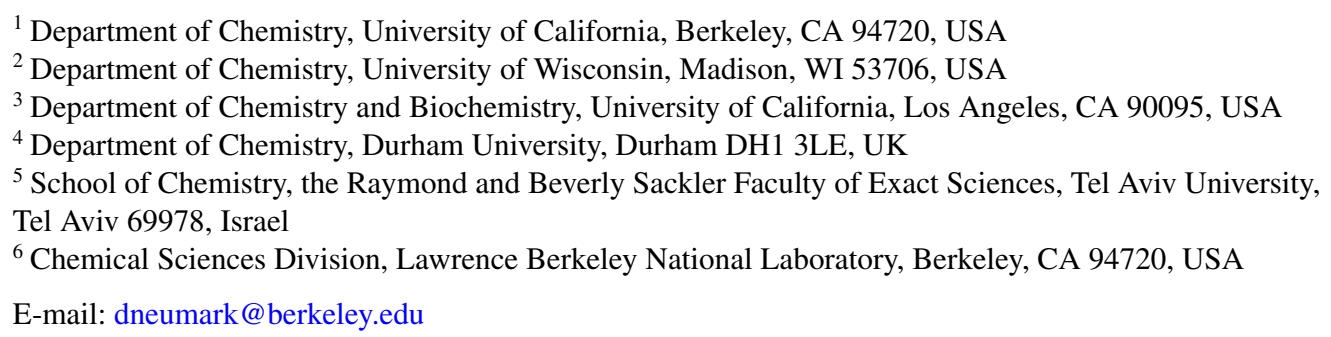

Received 24 February 2009

Accepted for publication 15 July 2009

Published 1 October 2009

Online at stacks.iop.org/PhysScr/80/048102

\begin{abstract}
We have examined size-dependent electronic relaxation dynamics in isolated semiconducting mercury cluster anions using time-resolved photoelectron imaging. Relaxation following excitation from within the conduction (p-) band occurs on an $\sim 3-40$ ps timescale and is attributed to non-adiabatic relaxation through the p-band. Exciting an electron from the valence (s-) band into the conduction band creates an electron-hole pair that relaxes prior to Auger emission. The dynamics associated with this feature occur on an $\sim 500 \mathrm{fs}$ timescale and are attributed to either a hole-induced contraction of the cluster or electron-electron scattering.
\end{abstract}

PACS numbers: $32.80 . \mathrm{Hd}, 33.60 .+\mathrm{q}, 36.40 . \mathrm{Wa}, 82.53 . \mathrm{Mj}$

(Some figures in this article are in colour only in the electronic version.)

\section{Introduction}

As nanotechnology becomes a more integral part of the broader technological landscape, it becomes increasingly important to gain a fundamental understanding of the electronic processes that occur at the nanoscale. For example, there has been considerable recent interest in the mechanisms of electronic relaxation in semiconductor quantum dots owing to the possible application of these species in solar cells [1]. Much of this interest has focused on the creation and relaxation dynamics of multiple charge carriers in quantum dots, either by absorption of multiple photons by a single quantum dot or through the proposed mechanism of multiple exciton generation [2, 3], in which a high-energy electron-hole pair created by a single photon decays into multiple electron-hole pairs.
Quantum dots are generally formed in solution and passivated with surface-capping groups, however, and some experiments have suggested that the surface-capping can have significant effects on the electronic dynamics of the quantum dots $[4,5]$. Hence, studies of bare, gas-phase species that mimic the electronic structure of quantum dots can provide new perspectives into the relaxation dynamics that occur in these species. In this paper, we present recent experimental work [6-9] on mercury cluster anions using time-resolved photoelectron imaging (TRPEI) to monitor electronic relaxation dynamics after (independently) exciting electrons from either the conduction or valence band.

Mercury clusters are an ideal system to study in this respect. Upon aggregation of $\mathrm{Hg}$ atoms, the filled atomic $6 s$ and empty $6 \mathrm{p}$ atomic orbitals form quasi-continuous 


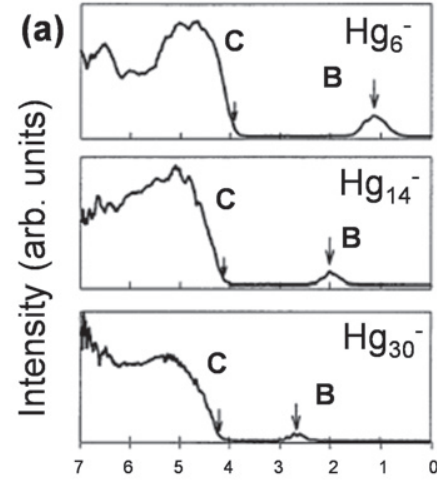

Electron Binding Energy (eV)

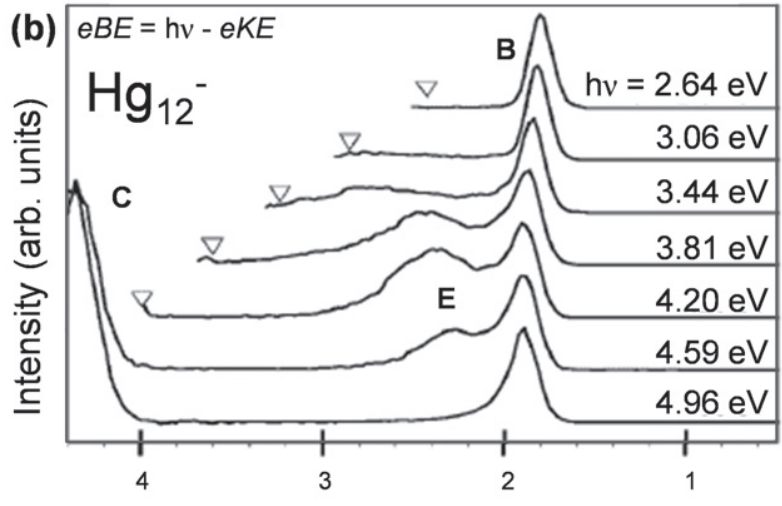

Electron Binding Energy (eV)

Figure 1. (a) Photoelectron spectra of $\mathrm{Hg}_{n}^{-}$measured with $7.9 \mathrm{eV}$ photons adapted from Busani et al [21]. The gap between the low electron binding energy (eBE) (6p electrons) peak and the broad, high eBE shoulder (6s electrons) decreases with cluster size. This corresponds to the $\mathrm{BG}$ of the neutral $\mathrm{Hg}_{n}$ cluster. (b) Photoelectron spectra measured using a tunable UV nanosecond laser, showing the $\mathrm{Hg}_{n}^{-}$BG and the wavelength-dependent appearance of a new feature due to delayed Auger emission, adapted from Busani $e t$ al [22]. The $e^{-}-h^{+}$pair could potentially relax much further (triangles) than the signal actually extends, indicating that there is some fast Auger emission that inhibits this process. See text for details.

'bands'7. These bands are well separated, having a distinct band gap (BG) that drops with increasing cluster size, similar to the situation in semiconductor quantum dots. Clusters of only a few $\mathrm{Hg}$ atoms will be bound in van der Waals (vdW) complexes [10] before making a transition to a more covalent binding character characteristic of semiconductors, with increased delocalization of electrons throughout the cluster. This change in bond character has been studied extensively in both neutral [11-16] and charged [17-20] clusters. Eventually, the addition of orbitals to the bands leads to the closure of the BG, in a Kubo-type insulator-to-metal transition [19].

Mercury cluster anions, $\mathrm{Hg}_{n}^{-}$, offer a natural way to probe the size evolution of these trends. Since the clusters are charged, size-selection prior to spectroscopic interrogation is straightforward. Moreover, in the cluster anions, the excess electron resides at the bottom of the p-band. Thus, as shown by Cheshnovsky and co-workers, photoelectron spectroscopy of $\mathrm{Hg}_{n}^{-}$anions at photon energies high enough to detach electrons from both the $\mathrm{p}$ - and s-bands can directly yield the $\mathrm{BG}$ as a function of cluster size [21-23]. Characteristic photoelectron spectra are shown in figure 1(a). Basic processes yielding these features are shown schematically on the left part of figure 2. In this case, direct UV detachment from the p- and s-bands (corresponding to Features B and C) yield the BG in the photoelectron spectrum. Extrapolation of extracted $\mathrm{BG}$ energies indicates that the transition to metallic bonding (i.e. $\mathrm{BG} \sim 0$ ) occurs around $n=400 \pm 30 \mathrm{Hg}$ atoms.

An interesting effect in the photoelectron spectra of $\mathrm{Hg}_{n}^{-}$ anions is shown in figure 1(b), where photoelectron spectra for $\mathrm{Hg}_{12}^{-}$are shown as a function of photon energy. In addition to the direct detachment features $\mathrm{B}$ and $\mathrm{C}$, an additional feature (E) appears at slightly higher eBEs than feature B at selected photon energies. This results from resonant excitation

7 These are not 'bands' in the strictest sense, as there is always some energy spacing for finite systems, but for the sake of simplicity that term will be used throughout this work.

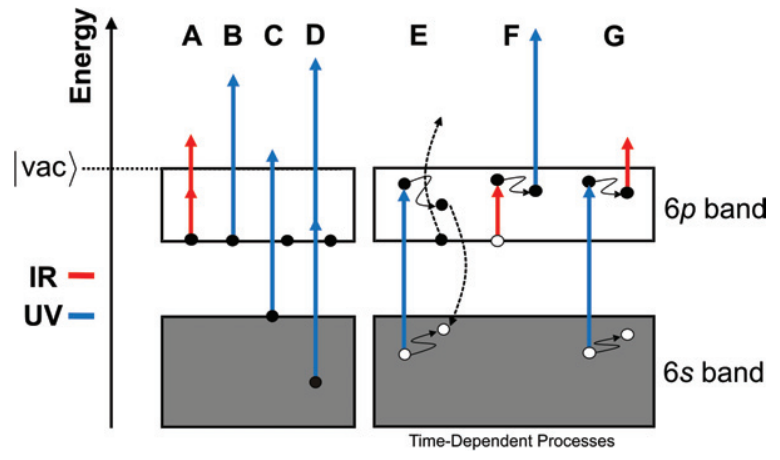

Figure 2. Schematic of possible photoinduced electronic processes in $\mathrm{Hg}_{n}^{-}$for the experiments described in the text. Processes A-D show no time dependence beyond cross correlation limited depletion effects. Process E describes Auger emission, which is detectable only after some relaxation of the excited electron or hole (or both); process $\mathrm{F}$ is the intraband excitation and relaxation prior to detachment; and process $\mathrm{G}$ describes the transient interband population. Red denotes IR wavelengths, blue denotes UV wavelengths. See text for exact combinations used in each experiment.

of an electron from the s-band to the p-band, creating an electron-hole $\left(e^{-}-h^{+}\right)$pair in the cluster. This $e^{-}-h^{+}$pair can decay by an Auger process, in which recombination releases enough energy to eject the excess electron originally lying at the bottom of the p-band (process E in figure 2). Because these electrons appear at lower electron kinetic energies (eKEs) than the direct detachment feature B, some relaxation of the nascent $e^{-}-h^{+}$pair must occur prior to Auger emission.

These observations raise questions about the rate of this Auger process and the nature of the relaxation prior to electron emission. We address these questions here using TRPEI. In these experiments, we use one femtosecond laser pulse to excite an electron within the cluster to a higher electronic state and then a second such pulse to detach the electron after a variable time-delay. We have monitored the dynamics following the two types of excitation: 'intraband' $\left(6 \mathrm{p} \rightarrow 6 \mathrm{p}^{*}\right)$ and 'interband' $\left(6 \mathrm{~s}^{2 n} 6 \mathrm{p}^{1} \rightarrow 6 \mathrm{~s}^{2 n-1} 6 \mathrm{p}^{1} 6 \mathrm{p}^{*}\right)$ excitation. 


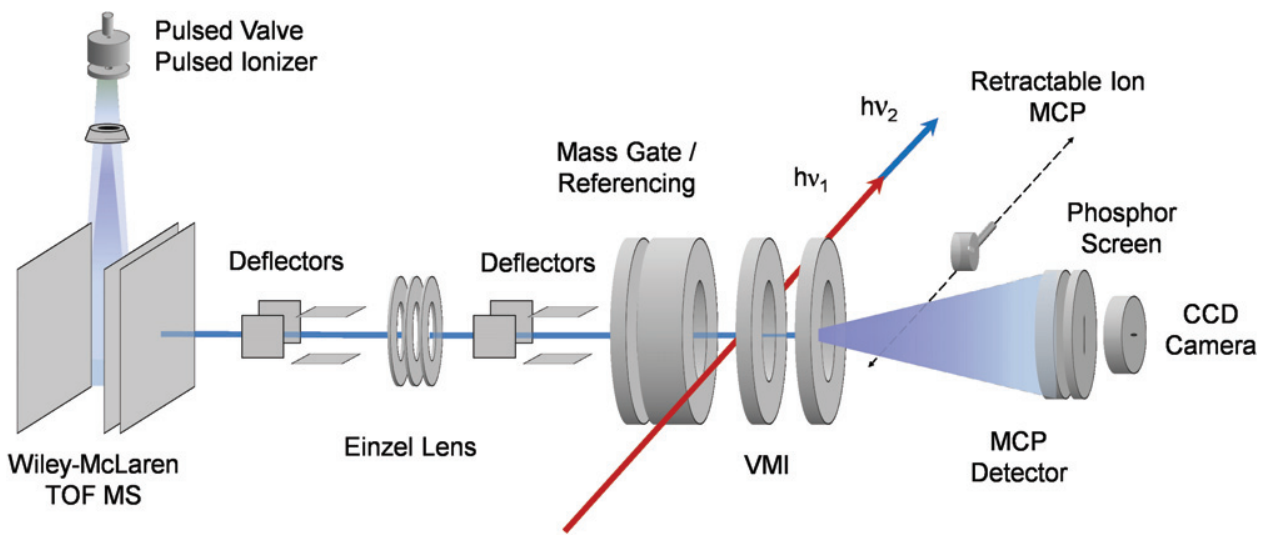

Figure 3. Anion TRPEI apparatus.

\section{Experimental set-up}

The experimental set-up for TRPEI has been described in detail elsewhere [24]; a brief summary is given here and the apparatus is shown schematically in figure 3. Mercury clusters are produced via a seeded supersonic expansion of argon and $\mathrm{Hg}$ vapor (over $230{ }^{\circ} \mathrm{C}$ liquid $\mathrm{Hg}$ ) into a vacuum chamber using a pulsed solenoid valve [25] and ionized with a pulsed ring electrode. Anions are then injected into a Wiley-McLaren [26] type time-of-flight (TOF) mass spectrometer. The cluster size of interest is isolated using a pulsed electrostatic switch. The size-selected ion packet is then crossed with femtosecond pump and probe laser pulses. The pump pulse electronically excites the anion, and at a designated time delay the probe pulse detaches electrons that are energetically accessible. Ejected photoelectrons are collected and analyzed by velocity-map imaging (VMI) [27] giving both velocity and angular information. The photoelectrons are accelerated toward a stack of microchannel plates (MCPs) coupled to a phosphor screen, creating an image that is captured in real time using a CCD camera. The raw images are four-way symmetrized to account for inhomogeneities in the detector, and then inverted using the BASEX transformation [28] to recover the three-dimensional photoelectron distribution. Angular integration yields the speed distribution of the electrons, from which we extract either the eKE or eBE distributions reported here $\left(e \mathrm{BE}=h v_{\text {probe }}-e \mathrm{KE}\right)$.

Laser pulses in these experiments are produced with a commercial Ti:sapphire femtosecond oscillator, amplified through regenerative chirped-pulse amplification (Clark-MXR NJA-5 and CPA-1000) to yield $\sim 100 \mathrm{fs}$ pulses with $1 \mathrm{~mJ}$ pulse energy at a fundamental wavelength of $\sim 800 \mathrm{~nm} / 1.55 \mathrm{eV}$. Second and third harmonics of this wavelength at $400 \mathrm{~nm} / 3.10 \mathrm{eV}$ and $266 \mathrm{~nm} / 4.65 \mathrm{eV}$, respectively, are generated in nonlinear BBO crystals, with typical pulse energies of $100 \mu \mathrm{J}$ pulse $^{-1}$. Infrared pulses at $1.0 \mathrm{eV}$ are generated using an optical parametric amplifier (TOPAS, Light-Conversion, Ltd), resulting in $\sim 1 \mu \mathrm{J}$ pulse $^{-1}$. The time resolution, given by the cross correlation of the pump and probe laser pulses, is $\sim 150 \mathrm{fs}$.

In the intraband experiments [6, 7], a pump pulse at either 1.55 or $1.0 \mathrm{eV}$ is used to excite an electron from the bottom of the p-band to a higher-lying $\mathrm{p}$-state and a $3.10 \mathrm{eV}$ probe pulse detaches at a prescribed time-delay. Interband experiments $[8,9]$ utilize a $4.65 \mathrm{eV}$ pump $/ 1.55 \mathrm{eV}$ probe scheme, where the UV pump excites an electron from the s-band into the p-band, and the $1.55 \mathrm{eV}$ probe pulse detaches electrons from the p-band.

\section{Dynamics in mercury cluster anions}

\subsection{Results}

Figure 4 shows representative photoelectron images and associated spectra for an intraband excitation experiment of $\mathrm{Hg}_{15}^{-}$at a pump energy of $1.55 \mathrm{eV}$ [6]. Peak $\mathrm{F}$ at $\sim 2.6 \mathrm{eV}$ at the zero-of-time delay $\left(t_{0}\right)$ in the progression results from detachment of the initially prepared excited state. At later time delays, the peak shifts to lower eKE until it almost coincides with the detachment of ground state electrons at the bottom of the p-band (feature B), at which point the dynamics are complete. For the cluster size range studied here $(n=11-16,18)$, these dynamics occur on a timescale of $\sim 10-40 \mathrm{ps}$. Because there are no other mobile charge carriers present, electronic relaxation can only occur via sequential radiationless transitions through the $\mathrm{p}$-band. Analogous experiments using $1.0 \mathrm{eV}$ pump photons [7] were conducted in similarly sized clusters $(n=7-13,15,18)$ and the resulting relaxation rates of $\sim 4-9$ ps were observed to be faster, on average, by a factor of 3-4.

A second set of experiments was performed to investigate the dynamics following interband excitation, pumping with $4.65 \mathrm{eV}$ and probing with $1.55 \mathrm{eV}$. Figure 5 shows time-resolved photoelectron spectra for $\mathrm{Hg}_{19}^{-}$over a 3 ps delay range. The integrated intensity of each feature is plotted as a false-color map over its respective energy range as a function of time delay. Features $\mathrm{A}, \mathrm{B}$ and $\mathrm{C}$ in figure 5 show only trivial depletion-related dynamics. These coincide with the same processes observed in the original one-color work of Cheshnovsky [21, 22], with the exception of feature A, which requires intensities associated with femtosecond lasers. However, the Auger feature E has time-dependence that extends well beyond the cross correlation of the pump and probe pulses, and is therefore of interest dynamically. In addition, there is another feature $(\mathrm{G})$ around $0.6 \mathrm{eV}$ that only appears in the time-resolved spectra. This feature appears in an energy range consistent with transient $\left[1+1^{\prime}\right]$ detachment 


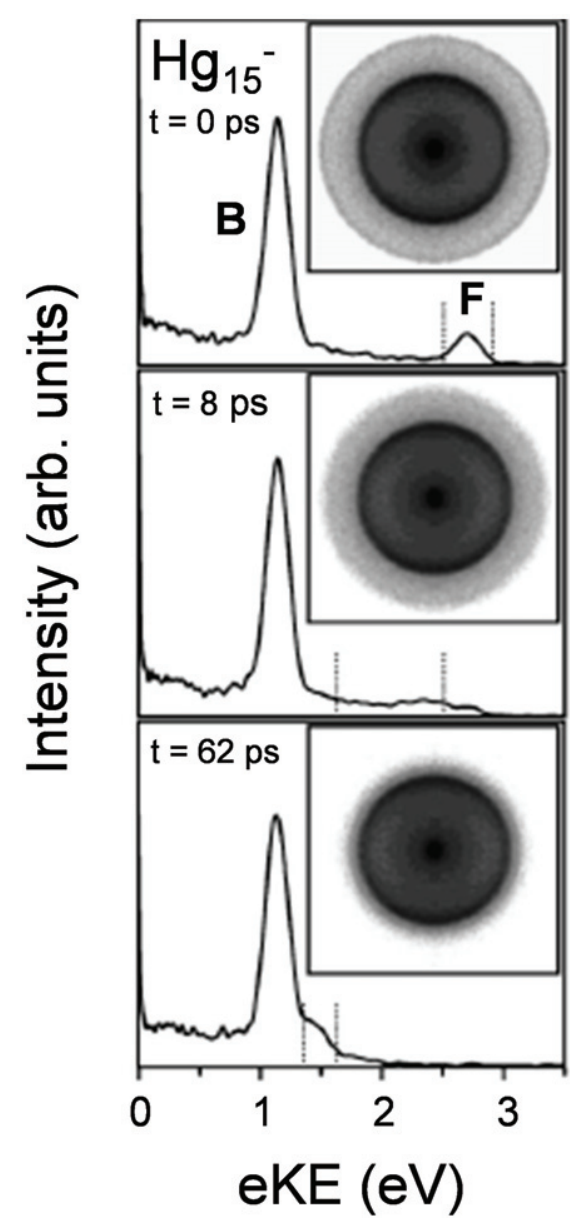

Figure 4. Photoelectron images and their associated spectra for $\mathrm{Hg}_{15}^{-}$at $t=0,8$, and $62 \mathrm{ps}$ following intraband excitation. The direct-detachment peak from the p-band (feature F) moves to lower eKE with increasing time delay as the excited electron non-adiabatically cascades through the states in the band [6].

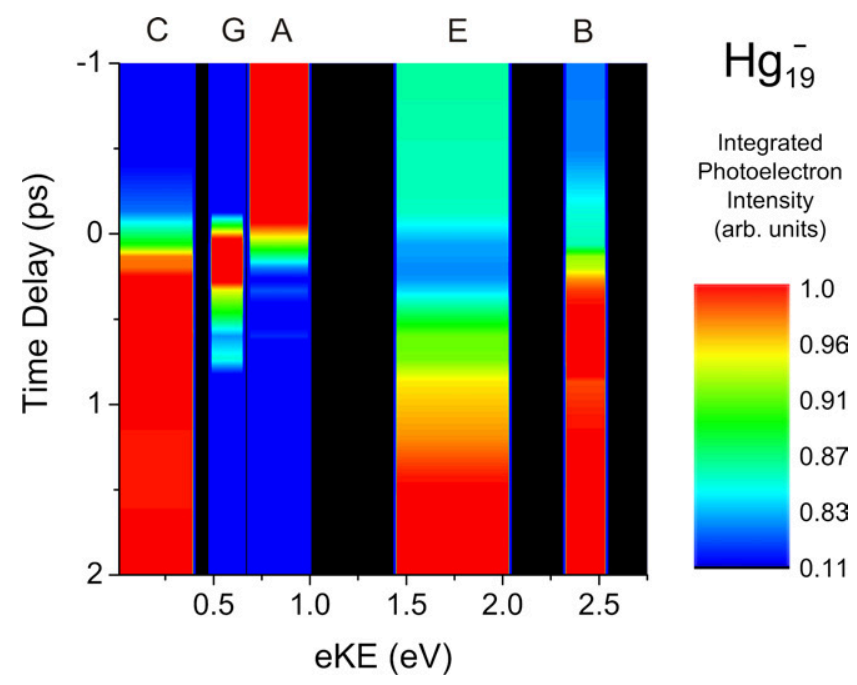

Figure 5. Time-resolved photoelectron spectra following interband excitation of $\mathrm{Hg}_{19}^{-}$. Each of the features is labeled according to the processes presented in figure 2. Feature $\mathrm{E}$ is the Auger tail described in the text and seen in previous one-color studies, seen here to evolve in time. Feature $\mathrm{G}$ at $\sim 0.6 \mathrm{eV}$ belongs to the transient interband electron population.

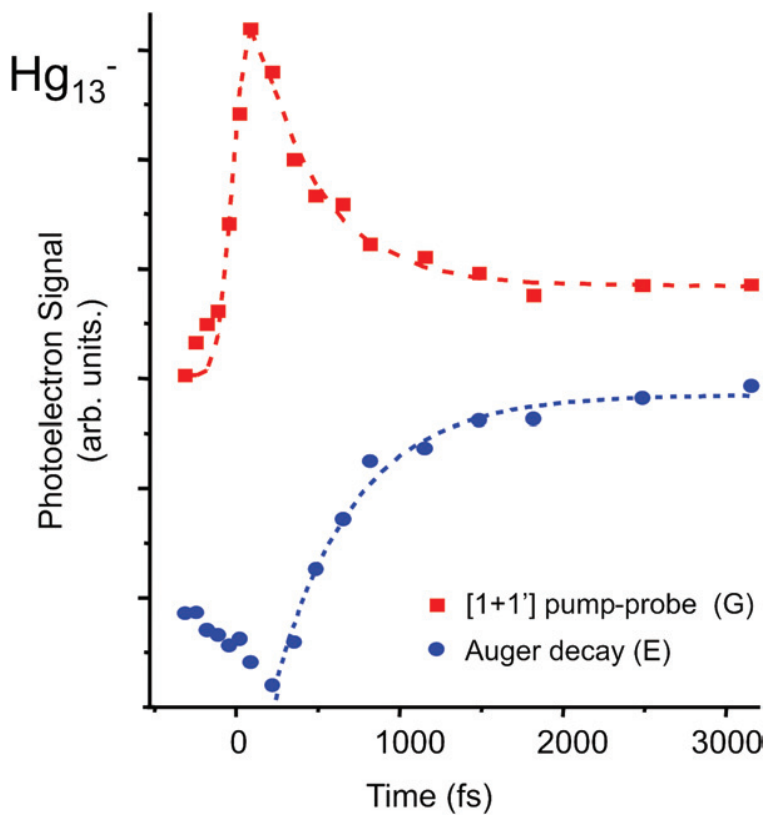

Figure 6. Integrated intensities of the Auger feature (E, blue) and the transient interband electron intensity $\left(\mathrm{G}\right.$, red) for $\mathrm{Hg}_{13}^{-}$. Feature $\mathrm{E}$ is fit to an exponential recovery with a time constant of $490 \pm 55 \mathrm{fs}$; feature $\mathrm{G}$ is fit to a convolution of a Gaussian (for the laser profile) and an exponential decay, with an extracted time constant of $400 \pm 55 \mathrm{fs}$

of excited electrons high enough in the p-band. It disappears slightly faster than the recovery of the Auger feature E, but lasts significantly longer than the cross correlation.

The integrated intensities of each of these features for $\mathrm{Hg}_{13}^{-}$are displayed in figure 6. The Auger emission signal is fit to an exponential recovery and occurs over approximately $490 \pm 55 \mathrm{fs}\left(\tau_{\mathrm{E}}\right)$. Changes in intensity before $t_{0}$ are due to depletion effects from the cross correlation of the two pulses, as at negative time-delays, the probe pulse arrives before the pump, depleting the excess electron (via channel A) prior to Auger ejection; these effects are discussed in detail in our previous work [8]. The transient p-state population must first be created by the pump pulse before being detached by the probe, thus the population is fit to a convolution of an exponential decay with a Gaussian with a width equal to the cross correlation time. The recovered time constant is $400 \pm$ $55 \mathrm{fs}\left(\tau_{\mathrm{G}}\right)$. Both processes show complementary dynamics: they occur over similar timescales and the recovery of Auger signal is concomitant with decay of the transient population. The complementary nature of the dynamics suggests that these features are probing the same dynamical process and fully account for the transient electron population.

Timescales for the intraband and interband experiments are shown as a function of cluster size in figure 7 . Notable features are that (a) intraband relaxation is faster for excitation at $1.0 \mathrm{eV}$ than at $1.55 \mathrm{eV}$, (b) relaxation subsequent to interband excitation is typically an order of magnitude faster than intraband relaxation and (c) the timescale for recovery of the Auger signal (feature E in figure 7(b)) rises abruptly at $n=13$. 


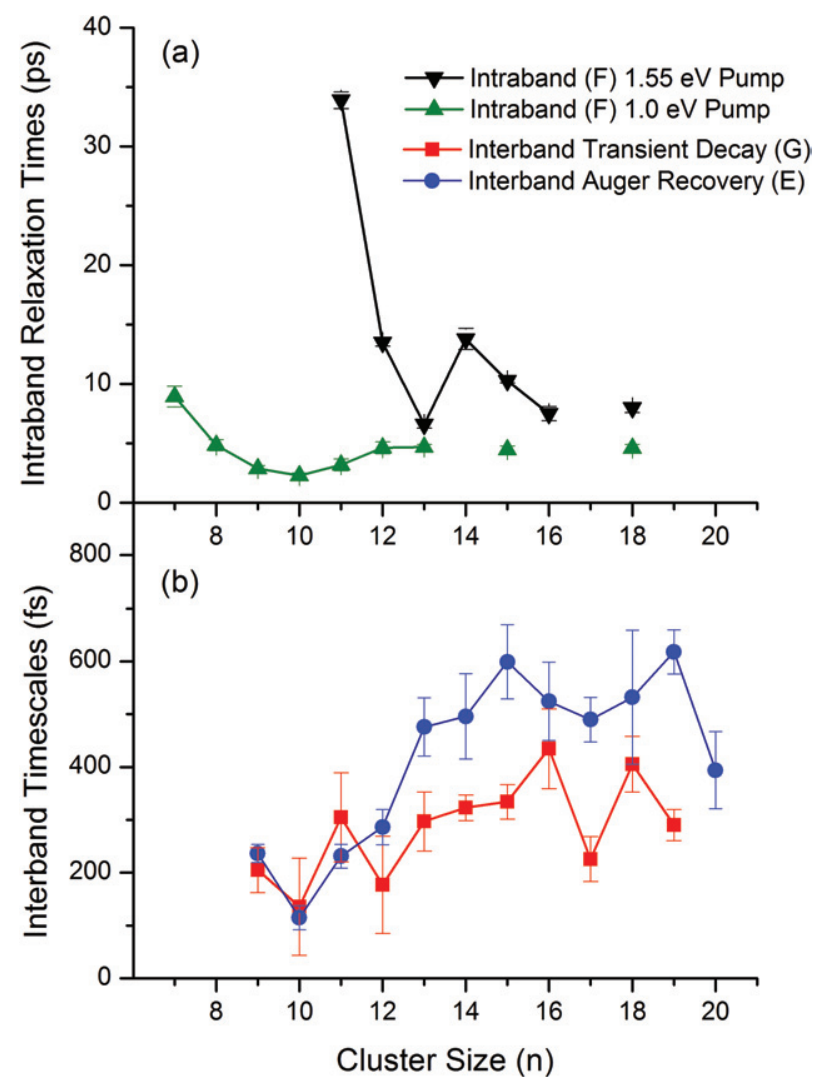

Figure 7. (a) Timescales for non-adiabatic relaxation through the p-band following intraband excitation $(1.55 \mathrm{eV}$ in black and $1.0 \mathrm{eV}$ in green) as a function of cluster size; this relaxation occurs on a $\sim 10$ ps timescale. (b) Auger emission timescales (feature $\mathrm{E}$ in blue) and transient interband electron decay timescales (feature $\mathrm{G}$ in red) as a function of cluster size, on a femtosecond timescale.

\subsection{Discussion}

In the case of intraband excitation, the lone electron at the bottom of the p-band is excited to a higher-lying p-state (process $\mathrm{F}$ in figure 2) using a low energy IR pump photon. Since there are no other charge carriers present in the conduction band, the electron can only relax via the cluster equivalent of electron-phonon coupling. The overall shape of the eKE progressions suggests that the electron relaxes from one electronic state to another, cascading non-adiabatically through the p-band, a mechanism reinforced by kinetic modeling with a simple master equation approach of the observed dynamics [6]. In this size range, the electronic states within the p-band are spaced by $\sim 0.1 \mathrm{eV}$ [10], and each non-adiabatic transition results in transfer of electronic energy into vibrational modes of the cluster.

The observed relaxation rates corresponding to the timescales reported in figure $7(a)(1 / \tau)$ generally increase with cluster size, but not in a simple monotonic fashion. In contrast, rates obtained from the described kinetic model (which does not incorporate any quantum effects and assumes equal coupling over a uniform density of states (DOS)) scale linearly [6]; each $\mathrm{Hg}$ atom contributes three unoccupied p-states, so the DOS (and thus the rate) should increase linearly with cluster size. Quantum and surface effects inherent to the molecularity and high fraction of surface atoms in this size range thus still have a strong influence on the relaxation rate. The somewhat faster relaxation at $1.0 \mathrm{eV}$ is rather surprising, since one generally expects more rapid relaxation for higher excitation energies, since more relaxation pathways are accessible.

A possible explanation for faster relaxation is that the electronic DOS is not uniform across the p-band, an idea that is supported by calculations [10] of neutral clusters. A higher DOS closer to the bottom of the p-band, for example, would require less energy being transferred into cluster vibrational modes for each radiationless transition, thereby increasing the relaxation rate. However, more detailed theory is needed to confirm this hypothesis.

From figure 7 , it can easily be seen that the recovery of the Auger (E) and the decay time of the $\left[1+1^{\prime}\right](\mathrm{G})$ features in the interband excitation experiments are significantly faster than the intraband relaxation at either pump energy. Both the Auger and transient timescales show an overall increase with cluster size, though there is also a high degree of variation over this small size range, again highlighting the molecular nature of these clusters. The faster dynamics subsequent to interband excitation reflects the presence of multiple charge carriers, i.e. two electrons and one hole, and the accompanying larger variety of decay channels accessible to the excited state created by the pump pulse. While the excited p-electron can decay by electronic $\rightarrow$ vibrational energy transfer in both cases, the Auger decay pathway is accessible only upon interband excitation. This situation is similar to that in quantum dots, in which excited states with multiple excitons decay much more rapidly than states with a single $e^{-}-h^{+}$pair [29, 30].

The marked jump in $\tau_{\mathrm{E}}$ toward longer lifetimes at $n=13$ coincides with various other observations in both experiments and calculations on neutral and charged $\mathrm{Hg}$ clusters, which indicate a transition from vdW to covalent bonding [10-19]. It should be noted that the $n=13$ geometry is predicted to be a $\mathrm{Hg}$ atom encapsulated by an icosahedral shell [14]. This, combined with the reduced surface area of such a symmetric structure and the heightened number of nearest neighbors would lead to an increase in sharing of the electrons and mixing/hybridization of the s- and p-orbitals. Also, the high symmetry of this shape will facilitate delocalization of the excess charge, which could explain why the transient times are less sensitive to the size change at $n \geqslant 13$.

As mentioned above, Auger emission occurs with lower eKE than the direct detachment feature, which means the $e^{-}-h^{+}$pair must relax before recombining. This relaxation must be due to something other than the stepwise non-adiabatic transitions inferred from the intraband experiments, as the Auger timescale is more than an order of magnitude faster than the timescales in figure 7(a). Calculations [15] using the diatomics-in-molecules (DIM) approach suggest that in neutral $\mathrm{Hg}$ clusters the creation of an $e^{-}-h^{+}$pair leads to a contraction of the nearest-neighbor bond lengths. This hole-induced contraction likely occurs in the anionic clusters as well. Such a process would have substantial effects on the relaxation dynamics, as the s-electron is now excited to a highly perturbed electronic state, far from the equilibrium bond distance at the minimum of the potential energy surface. The excited cluster can then undergo vibrational relaxation toward the equilibrium 
geometry, leading to dissipation along the nuclear coordinates such that that energy is no longer available to the electron.

Moreover, because there are two electrons in the p-band and one hole in the s-band in the interband experiments, the excited electron can relax by multiple pathways, moving lower in energy through the manifold of anion excited states, with the energy difference being made up via inelastic electron-electron and/or electron-hole scattering. Indeed, electron-electron scattering has been invoked to explain the rapid timescales seen for ultrafast electron relaxation in transition metal clusters as well [31-33]. Here, the manifold of anion states is much denser than that belonging to those excited states where there is only one p-band electron and no hole in the s-band; relaxation along this manifold can also occur as the hole rises in energy. Through either mechanism, the emitted Auger electron has less energy available to it than before relaxation, and thus will be detected at lower kinetic energy, which must occur before the electrons are emitted. Thus the timescales extracted from each process represent upper bounds to the actual relaxation times, which, again, are an order of magnitude faster than the non-adiabatic relaxation of a single electron in the p-band seen in the intraband excitation experiments.

\section{Outlook}

Our extensive experiments have determined the timescales of various electronic relaxation processes in isolated semiconducting clusters. A single mobile electron within the conduction band will relax via interactions with the phonon bath over tens of picoseconds as it falls through the energy levels of the conduction band. However, the presence of additional charge carriers is seen to have a dramatic effect on the decay of the electronic excitation. A change in geometry induced by a hole in the valence band and/or electron-electron scattering causes a substantial increase in the rate of energy transfer, into the femtosecond regime.

At present, however, it is unclear whether it is the electron or the hole, or both in conjunction that are responsible for the relaxation in $\mathrm{Hg}_{n}^{-}$. The analogous process creating the $e^{-}-h^{+}$ pair from the d-band could elucidate the role of the hole in the overall relaxation. The density of p-states would remain the same but the density of hole states would presumably be much larger and thus the hole could relax much faster, increasing the rate of Auger decay.

Investigating both the intra- and interband excitation studies over a broader size range is the next natural step, as the clusters become more similar to their quantum dot analogues and structural differences between anions and neutrals should be reduced. Figure 7 seems to suggest convergence toward a large-size limit for the timescales of the processes involved, at least over the size range of the vdW-to-covalent transition. At much larger cluster sizes, the effect of the insulator-to-metal transition on the relaxation rates would also be of considerable interest. Experiments to probe these effects are planned in our laboratory.

Varying the pump energy should also yield interesting results. In addition to being able to access the d-band electrons in larger clusters, pumping with higher frequency light could generate multiple excitons through charge-carrier multiplication $[2,3]$. The effect on the observable relaxation rates would yield more insight into the nature of the relaxation and provide a clear parallel to studies on larger quantum dots.

Central to all of these experiments is a detailed knowledge of the band structure. An explicit mapping of the DOS with higher energy resolution over the cluster size regime studied would aid in the interpretation of these results, especially for the pump energy-dependent intraband relaxation rates. This information could be obtained through a combination of experiment and improved electronic structure calculations. In addition, experiments that probe the geometric evolution of neutral and charged mercury clusters with size would be exceedingly valuable in understanding the trends seen here. Electron diffraction experiments on size-selected clusters [34] may be very useful in addressing this issue.

\section{Acknowledgments}

This work was supported by the National Science Foundation (CHE-0649647) and the United States-Israel Binational Science Foundation (2000-333 and 2004-401). OTE acknowledges the Alexander von Humboldt Foundation for a Feodor-Lynen fellowship.

\section{References}

[1] Huynh W U, Dittmer J J and Alivisatos A P 2002 Science 295 2425

[2] Schaller R D, Agranovich V M and Klimov V I 2005 Nat. Phys. 1189

[3] Ellingson R J, Beard M C, Johnson J C, Yu P, Micic O I, Nozik A J, Shabaev A and Efros A L 2005 Nano Lett. 5865

[4] Guyot-Sionnest P, Wehrenberg B and Yu D 2005 J. Chem. Phys. 123074709

[5] Pandey A and Guyot-Sionnest P 2007 J. Chem. Phys. 127 111104

[6] Verlet J R R, Bragg A E, Kammrath A, Cheshnovsky O and Neumark D M 2004 J. Chem. Phys. 12110015

[7] Bragg A E, Verlet J R R, Kammrath A, Cheshnovsky O and Neumark D M 2005 J. Chem. Phys. 122054314

[8] Griffin G B, Kammrath A, Ehrler O T, Young R M, Cheshnovsky O and Neumark D M 2008 Chem. Phys. 35069

[9] Griffin G B, Ehrler O T, Kammrath A, Young R M, Cheshnovsky O and Neumark D M 2009 J. Chem. Phys. 130231103

[10] Pastor G M and Bennemann K H 1994 Clusters of Atoms and Molecules ed H Haberland (Berlin: Springer) p 86

[11] Rademann K, Kaiser B, Even U and Hensel F 1987 Phys. Rev. Lett. $\mathbf{5 9} 2319$

[12] Haberland H, Kornmeier H, Langosch H, Oschwald M and Tanner G 1990 J. Chem. Soc. Faraday Trans. 862473

[13] Brechignac C, Broyer M, Cahuzac P, Delacretaz G, Labastie P, Wolf J P and Woste L 1988 Phys. Rev. Lett. 60275

[14] Moyano G E, Wesendrup R, Sohnel T and Schwerdtfeger P 2002 Phys. Rev. Lett. 89103401

[15] Kitamura H 2007 Eur. Phys. J. D 4333

[16] Kitamura H 2006 Chem. Phys. Lett. 425205

[17] Gaston N, Schwerdtfeger P and von Issendorf B 2006 Phys. Rev. A 74043203

[18] Haberland H, von Issendorf B, Yufeng J and Kolar T 1992 Phys. Rev. Lett. 693212

[19] von Issendorf B and Cheshnovsky O 2005 Annu. Rev. Phys. Chem. 56549

[20] Wang Y, Flad H and Dolg M 2000 Int. J. Mass Spectrom. 201 197 
[21] Busani R, Folkers M and Cheshnovsky O 1998 Phys. Rev. Lett. 813836

[22] Busani R, Giniger R, Hippler T and Cheshnovsky O 2003 Phys. Rev. Lett. 90083401

[23] Busani R and Cheshnovsky O 2007 J. Phys. Chem. C 111 17725

[24] Davis A V, Wester R, Bragg A E and Neumark D M 2003 J. Chem. Phys. 118999

[25] Even U, Jortner J, Noy D, Lavie N and Cossart-Magos C 2000 J. Chem. Phys. 1128068

[26] Wiley W C and McLaren I H 1955 Rev. Sci. Instrum. 26 1150

[27] Eppink A T J B and Parker D H 1997 Rev. Sci. Instrum. 68 3477
[28] Dribinski V, Ossadtchi A, Mandelshtam V A and Reisler H 2002 Rev. Sci. Instrum. 732634

[29] Efros A L, Kharchenko V A and Rosen M 1995 Solid State Commun. 93281

[30] Klimov V I, Mikhailovsky A A, McBranch D W, Leatherdale C A and Bawendi M G 2000 Science 2871011

[31] Knoesel E, Hotzel A and Wolf M 1998 Phys. Rev. B 5712812

[32] Pontius N, Neeb M and Eberhardt W 2003 Phys. Rev. B 67 035425

[33] Pontius N, Bechthold P S, Need M and Eberhardt W 2000 Phys. Rev. Lett. 841132

[34] Oger E, Kelting R, Weis P, Lechtken A, Schooss D, Crawford N R M, Ahlrichs R and Kappes M M 2009 J. Chem. Phys. 130124305 